\title{
Numerical solutions and error estimations for the space fractional diffusion equation with variable coefficients via Fibonacci collocation method
}

\author{
Ayşe Kurt Bahşı and Salih Yalçınbaş ${ }^{*}$ (i)
}

\author{
${ }^{*}$ Correspondence: salih. \\ yalcinbas@cbu.edu.tr \\ Department of Mathematics, \\ Manisa Celal Bayar University, \\ Manisa, Turkey
}

\begin{abstract}
In this study, the Fibonacci collocation method based on the Fibonacci polynomials are presented to solve for the fractional diffusion equations with variable coefficients. The fractional derivatives are described in the Caputo sense. This method is derived by expanding the approximate solution with Fibonacci polynomials. Using this method of the fractional derivative this equation can be reduced to a set of linear algebraic equations. Also, an error estimation algorithm which is based on the residual functions is presented for this method. The approximate solutions are improved by using this error estimation algorithm. If the exact solution of the problem is not known, the absolute error function of the problems can be approximately computed by using the Fibonacci polynomial solution. By using this error estimation function, we can find improved solutions which are more efficient than direct numerical solutions. Numerical examples, figures, tables are comparisons have been presented to show efficiency and usable of proposed method.
\end{abstract}

Keywords: Fibonacci polynomials, Fractional diffusion equations, Collocation method, Matrix relations, Error estimation algorithm

\section{Background}

In recent years, many studies have been developed about fractional partial differential equations. Fractional partial differential equations arise in many physical and engineering problems such as fractional order diffusion equations which are generalizations of classical diffusion equations and modelling of linear vibrations of axial moving systems (Saadatmandi and Dehghan 2011; Dönmez Demir et al. 2013).

In this paper, we consider the one-dimensional space fractional diffusion equation with variable coefficients

$$
\frac{\partial u(x, t)}{\partial t}=c(x) \frac{\partial^{\alpha} u(x, t)}{\partial x^{\alpha}}+q(x, t), \quad 0<x<l, \quad 0 \leq t \leq \tau, \quad 1<\alpha \leq 2,
$$

with the initial condition

$$
u(x, 0)=k(x), \quad 0<x<l,
$$

(c) 2016 The Author(s). This article is distributed under the terms of the Creative Commons Attribution 4.0 International License (http://creativecommons.org/licenses/by/4.0/), which permits unrestricted use, distribution, and reproduction in any medium, provided you give appropriate credit to the original author(s) and the source, provide a link to the Creative Commons license, and indicate if changes were made. 
and the boundary conditions

$$
\begin{aligned}
u(0, t)=g_{0}(t), & 0<t \leq \tau, \\
u(l, t)=g_{1}(t), & 0<t \leq \tau,
\end{aligned}
$$

where $c(x) \neq 0$ is diffusion coefficient, $q(x, t), k(x), g_{0}(t)$ and $g_{1}(t)$ are known functions and the function $u(x, t)$ is unknown.

Some authors interested in the numerical solution of Eq. (1) by using various methods such as Tau method (Saadatmandi and Dehghan 2011), Chebyshev collocation method (Khader 2011), spatial extrapolation (Tadjeran et al. 2006) and finite difference method (Dehghan 2006).

\section{Fibonacci polynomials}

In this study, we introduce Fibonacci collocation method based on matrix relations which has been used to find the approximate solutions of some classes of the differential equations such as integro-differential equations, differential-difference equations, Fredholm integro differential-difference equations, Pantograph-type functional differential equations and linear Volterra integro differential equations. Fibonacci collocation method is presented for the solution of mth-order linear differential-difference equations with variable coefficients under the mixed conditions (Kurt et al. 2013a, b) and this method is also used to solve both the linear Fredholm integro-differential-difference equations (Kurt et al. 2013a, b) and high-order Pantograph-type functional differential equations (Kurt Bahşı et al. 2015). On the other hand this method is applied for the linear Volterra integro differential equations (Kurt Bahşı and Yalçınbaş 2016a, b). Finally the mentioned method is presented for the class of the partial differential equations which are called Telegraph equations (Kurt Bahşı and Yalçınbaş 2016a, b). Also recently there are several using the other collocation methods to solve different types of the partial differential equations by using various special polynomials without Fibonacci polynomials such as Taylor (Bülbül and Sezer 2011), Bessel (Yüzbaşı and Şahin 2013), Chebyshev (Yüksel and Sezer 2014), Bernstein (Isik et al. 2014) and Bernoulli (Erdem Biçer and Yalçinbas 2016). On the other hand, these matrix methods were used for the numerical analysis of the longitudinal vibration of rods (Çevik 2010). By developing the Fibonacci collocation method, we will obtain the approximate solution of Eq. (1) in the truncated Fibonacci series form

$$
u_{N}(x, t)=\sum_{m=1}^{N} \sum_{n=1}^{N} a_{m n} F_{m}(x) F_{n}(t)
$$

where $a_{m n} ; m, n=1, \ldots, N$ are the unknown Fibonacci coefficients and $F_{n}(x), F_{m}(x)$; $m, n=1, \ldots, N$ are the Fibonacci functions defined by,

$$
F_{n}(x)=\sum_{j=0}^{\left[\frac{(n-1)}{2}\right]}\left(\begin{array}{c}
n-j-1 \\
j
\end{array}\right) x^{n-2 j-1}, \quad[(n-1) / 2]=\left\{\begin{array}{cc}
\frac{n-2}{2}, & n \text { even } \\
\frac{n-1}{2}, & n \text { odd }
\end{array}\right.
$$




\section{The fractional derivative in the Caputo sense}

Definition 1 Caputo's definition of the fractional-order derivative is defined as

$$
D^{\alpha} f(x)=\frac{1}{\Gamma(n-\alpha)} \int_{0}^{x} \frac{f^{(n)}(t)}{(x-t)^{\alpha+1-n}} d t, \quad n-1<\alpha<n, \quad n \in \mathbb{N}
$$

where $\alpha>0$ is the order of derivative, $\Gamma($.$) is the Gamma function and n=[\alpha]+1$, with $[\alpha]$ denoting the integral part of $\alpha$.

Recall that for $\alpha \in \mathbb{N}$, the Caputo differential operator coincides with the usual differential operator of integer order. For the Caputo's derivative we have (Diethelm 2010),

$$
\begin{aligned}
D^{\alpha} C & =0, \quad(C \text { is a constant }) \\
D^{\alpha} x^{\beta} & = \begin{cases}0, & \text { for } \beta \in \mathbb{N}_{0} \text { and } \beta<[\alpha] . \\
\frac{\Gamma(\beta+1)}{\Gamma(\beta+1-\alpha)}, & \text { for } \beta \in \mathbb{N}_{0} \text { and } \beta \notin \mathbb{N} \text { and }\end{cases}
\end{aligned}
$$

We use the ceiling function $[\alpha]$ to denote the smallest integer greater than or equal to $\alpha$, the floor function $[\alpha]$ to denote the largest integer less than or equal to $\alpha$ and $\beta>[\alpha]$ to denote order of $x$.

We use also $\mathbb{N}=\{1,2, \ldots\}$ and $\mathbb{N}_{0}=\{0,1,2, \ldots\}$. Similar to integer-order differentiation, Caputo's fractional differentiation is a linear operator.

\section{Fundamental matrix relations}

In this part, we have given some fundamental matrix relations for transforming Eq. (1) to matrix equation forms.

To obtain the numerical solution of the one-dimensional space fractional diffusion problem by using Fibonacci polynomials, we first evaluate the Fibonacci coefficients of the unknown function. The approximate solution (2) can be written in the matrix form

$$
u_{N}(x, t)=\mathbf{F}(x) \overline{\mathbf{F}}(t) \mathbf{A}
$$

where

$$
\begin{aligned}
\mathbf{F}(x) & =\left[\begin{array}{cccc}
F_{1}(x) & F_{2}(x) & \cdots & F_{N}(x)
\end{array}\right]_{1 \times N}, \\
\overline{\mathbf{F}}(t) & =\left[\begin{array}{cccc}
\mathbf{F}(t) & \mathbf{0} & \cdots & \mathbf{0} \\
\mathbf{0} & \mathbf{F}(t) & \cdots & \mathbf{0} \\
\vdots & \vdots & \ddots & \vdots \\
\mathbf{0} & \mathbf{0} & \cdots & \mathbf{F}(t)
\end{array}\right]_{N \times N^{2}},
\end{aligned}
$$

and

$$
\mathbf{A}=\left[\begin{array}{llll}
\mathbf{A}_{1} & \mathbf{A}_{2} & \cdots & \mathbf{A}_{N}
\end{array}\right]_{1 \times N^{2}}^{T}
$$

such that

$$
\mathbf{A}_{i}=\left[\begin{array}{llll}
a_{i 1} & a_{i 2} & \cdots & a_{i N}
\end{array}\right]_{1 \times N}^{T}, \quad i=1,2, \ldots, N .
$$


In here, the matrix form $\mathbf{F}(x)$ can be written as

$$
\mathbf{F}(x)=\mathbf{X}(x) \mathbf{C}^{T}
$$

so that

$$
\mathbf{X}(x)=\left[\begin{array}{llll}
1 & x & \cdots & x^{N-1}
\end{array}\right] .
$$

If $N$ is even,

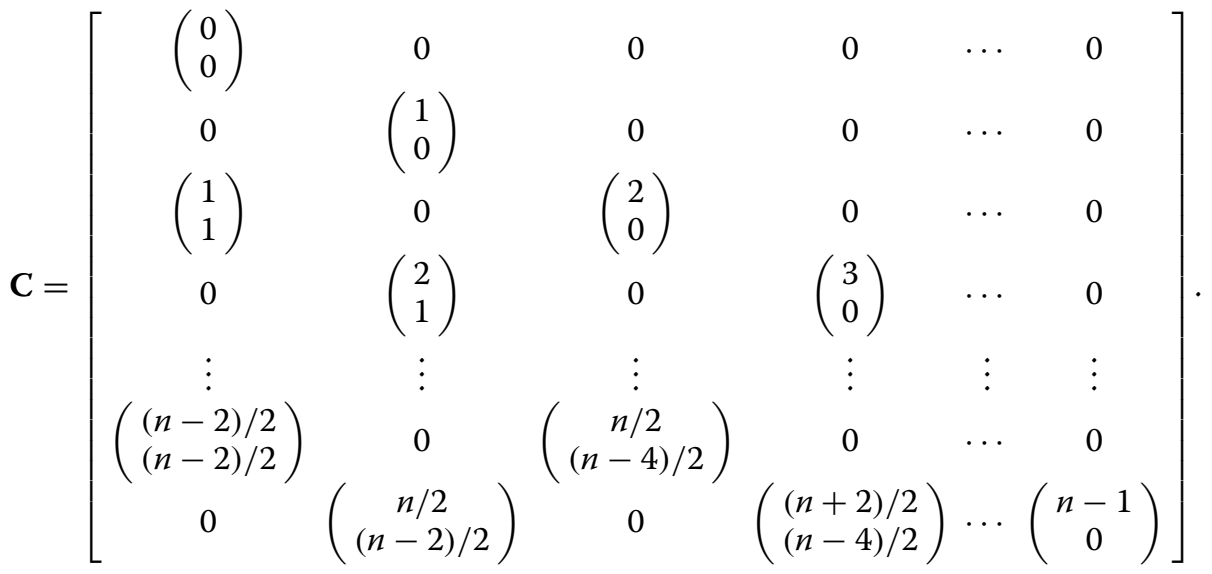

If $N$ is odd,

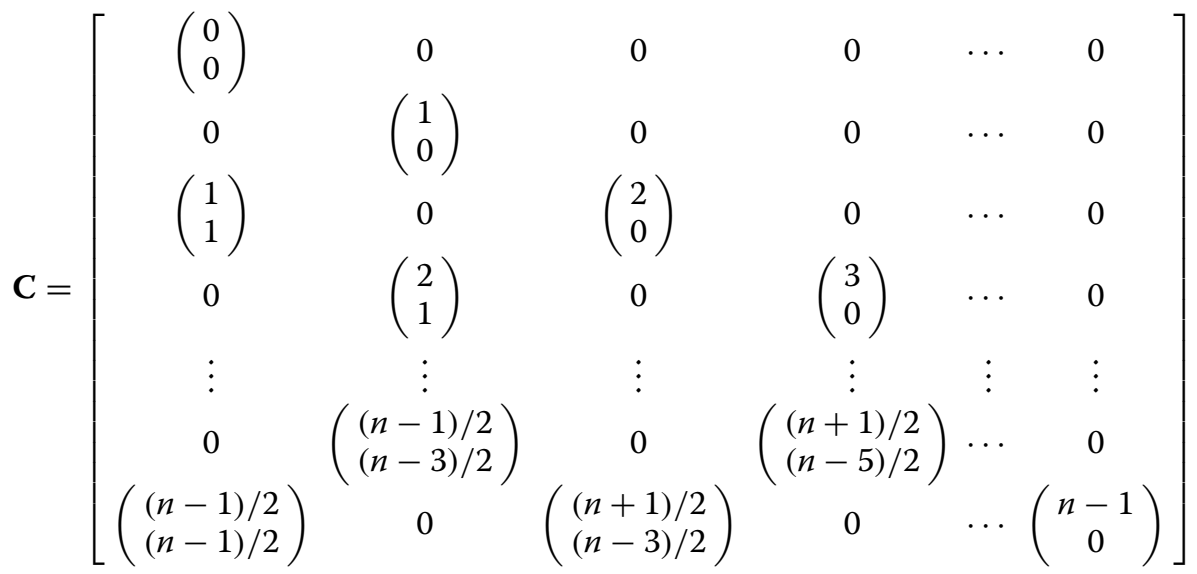

C is the characteristic matrix of the matrix relations (Kurt et al. 2013a, b). On the other hand, we can express the relations

$$
\overline{\mathbf{F}}(t)=\overline{\mathbf{X}}(t) \overline{\mathbf{C}}^{T}
$$

where

$$
\overline{\mathbf{X}}(t)=\left[\begin{array}{cccc}
\mathbf{X}(t) & \mathbf{0} & \cdots & \mathbf{0} \\
\mathbf{0} & \mathbf{X}(t) & \cdots & \mathbf{0} \\
\vdots & \vdots & \ddots & \vdots \\
\mathbf{0} & \cdots & \mathbf{0} & \mathbf{X}(t)
\end{array}\right]
$$


and

$$
\overline{\mathbf{C}}^{T}=\left[\begin{array}{cccc}
\mathbf{C}^{T} & \mathbf{0} & \cdots & \mathbf{0} \\
\mathbf{0} & \mathbf{C}^{T} & \cdots & \mathbf{0} \\
\vdots & \vdots & \ddots & \vdots \\
\mathbf{0} & \mathbf{0} & \cdots & \mathbf{C}^{T}
\end{array}\right]
$$

Firstly from the relations (3-5), we can obtain the desired solution $u(x, t)$ of Eq. (1) defined by the truncated Fibonacci series (2) in matrix form as follow

$$
u(x, t)=\mathbf{X}(x) \mathbf{C}^{T} \overline{\mathbf{X}}(t) \overline{\mathbf{C}}^{T} \mathbf{A}
$$

And secondly we can define the matrix form of the partial derivatives for each independent variables of $u(x, t)$ term can be written as

$$
\begin{aligned}
& u_{x}(x, t)=\mathbf{X}(x) \mathbf{B} \mathbf{C}^{T} \overline{\mathbf{X}}(t) \overline{\mathbf{C}}^{T} \mathbf{A} \\
& u_{t}(x, t)=\mathbf{X}(x) \mathbf{C}^{T} \overline{\mathbf{X}}(t) \overline{\boldsymbol{B}} \overline{\mathbf{C}}^{T} \mathbf{A}
\end{aligned}
$$

where

$$
\mathbf{B}=\left[\begin{array}{ccccc}
0 & 1 & 0 & \ldots & 0 \\
0 & 0 & 2 & & 0 \\
& \vdots & & \ddots & \vdots \\
0 & 0 & 0 & \ldots & N-1 \\
0 & 0 & 0 & & 0
\end{array}\right]
$$

and

$$
\overline{\mathbf{B}}=\left[\begin{array}{cccc}
\mathbf{B} & \mathbf{0} & \cdots & \mathbf{0} \\
\mathbf{0} & \mathbf{B} & \cdots & \mathbf{0} \\
\vdots & \vdots & \ddots & \vdots \\
\mathbf{0} & \mathbf{0} & \cdots & \mathbf{B}
\end{array}\right]
$$

We introduce the relation between the matrix $\mathbf{X}(x)$ and its derivatives $\mathbf{X}^{\prime}(x)$ which can be expressed as

$$
\mathbf{X}^{\prime}(x)=\mathbf{X}(x) \mathbf{B}
$$

Similarly, the relation between the matrix $\mathbf{X}(t)$ and its derivative $\mathbf{X}^{\prime}(t)$ is written

$$
\overline{\mathbf{X}}^{\prime}(t)=\overline{\mathbf{X}}(t) \overline{\mathbf{B}}
$$

Finally, we can explain the matrix form of $\frac{\partial^{\alpha} u(x, t)}{\partial x^{\alpha}}$ which is the fractional derivative of $u(x, t)$ term, can be written as

$$
\left[\frac{\partial^{\alpha} u(x, t)}{\partial x^{\alpha}}\right]=\mathbf{M}(x) \mathbf{C}^{T} \overline{\mathbf{X}}(t) \overline{\mathbf{C}}^{T} \mathbf{A}
$$


where

$$
\mathbf{M}(x)=\frac{\partial^{\alpha} \mathbf{X}(x)}{\partial x^{\alpha}}=\left[\begin{array}{lllll}
0 & D^{\alpha} x D^{\alpha} x^{2} & \cdots & D^{\alpha} x^{N-1}
\end{array}\right]_{1 \times N}
$$

such that

$$
D^{\alpha} x^{i}=\frac{\Gamma(i+1)}{\Gamma(i+1-\alpha)} x^{i-\alpha}, \quad i=1, \ldots, N-1 .
$$

By using the relations (7), (8), (9) into Eq. (1), we can have the closed matrix form

$$
\underbrace{\mathbf{X}(x) \mathbf{C}^{T} \overline{\mathbf{X}}(t) \overline{\mathbf{B}} \overline{\mathbf{C}}^{T}-c(x) \mathbf{M}(x) \mathbf{C}^{T} \overline{\mathbf{X}}(t) \overline{\mathbf{C}}^{T} \mathbf{A}}_{\mathbf{W}(x, t)}=q(x, t) .
$$

Briefly form is written

$$
\mathbf{W}(x, t) \mathbf{A}=q(x, t) .
$$

We have the corresponding matrix forms for the initial condition and for the boundary conditions (1) by means of the relation (6)

$$
\begin{aligned}
u(x, 0) & =\mathbf{X}(x) \mathbf{C}^{T} \overline{\mathbf{X}}(0) \overline{\mathbf{C}}^{T} \mathbf{A}=k(x) \\
u(0, t) & =\mathbf{X}(0) \mathbf{C}^{T} \overline{\mathbf{X}}(t) \overline{\mathbf{C}}^{T} \mathbf{A}=g_{0}(t) \\
u(l, t) & =\mathbf{X}(l) \mathbf{C}^{T} \overline{\mathbf{X}}(t) \overline{\mathbf{C}}^{T} \mathbf{A}=g_{1}(t)
\end{aligned}
$$

\section{Collocation method}

For the matrix equation Eq. (10) by using collocation points defined by

$$
\begin{aligned}
x_{i} & =\frac{l}{N-1}(i-1), \quad i=1,2, \ldots, N \\
t_{j} & =\frac{\tau}{N-1}(j-1), \quad j=1,2, \ldots, N .
\end{aligned}
$$

We obtain the system of matrix equations

$$
\mathbf{W}\left(x_{i}, t_{j}\right) \mathbf{A}=q\left(x_{i}, t_{j}\right), \quad i=1,2, \ldots, N, \quad j=1,2, \ldots, N .
$$

Using the system of matrix equation, the fundamental matrix equation becomes

$$
\mathbf{W A}=\mathbf{Q} \quad \text { or }[\mathbf{W} ; \mathbf{Q}]
$$

The fundamental matrix Eq. (4) of Eq. (1) corresponds to a system of $N^{2}$ algebraic equations for the $N^{2}$ unknown coefficients $a_{m n} ; m, n=1,2, \ldots, N$. On the other hand, by using collocation points, we can obtain matrix form of the initial condition

$$
\begin{aligned}
& \mathbf{U}_{1} \mathbf{A}=\mathbf{K} \\
& \mathbf{U}_{1}=\left[\mathbf{X}(x) \mathbf{C}^{\boldsymbol{T}} \overline{\mathbf{X}}(0) \overline{\mathbf{C}}^{\boldsymbol{T}}\right], \quad \mathbf{K}=\left[k\left(x_{i}\right)\right]_{N \times 1}, \quad i=1,2, \ldots, N
\end{aligned}
$$

and matrix form of the boundary conditions as follow

$$
\begin{aligned}
& \mathbf{U}_{2} \mathbf{A}=\mathbf{G}_{0} \\
& \mathbf{U}_{2}=\left[\mathbf{X}(0) \mathbf{C}^{\boldsymbol{T}} \overline{\mathbf{X}}(t) \overline{\mathbf{C}}\right], \quad \mathbf{G}_{0}=\left[g_{0}\left(t_{j}\right)\right]_{N \times 1}, \quad j=1,2, \ldots, N
\end{aligned}
$$


and

$$
\begin{aligned}
& \mathbf{U}_{3} \mathbf{A}=\mathbf{G}_{1} \\
& \mathbf{U}_{3}=\left[\mathbf{X}(l) \mathbf{C}^{\boldsymbol{T}} \overline{\mathbf{X}}(t) \overline{\mathbf{C}}^{\boldsymbol{T}}\right], \quad \mathbf{G}_{1}=\left[g_{1}\left(t_{j}\right)\right]_{N \times 1}, \quad j=1,2, \ldots, N .
\end{aligned}
$$

Subsequently, in order to obtain the solution of Eq. (1) under the initial and boundary conditions we can write augmented matrix which is contain all components of this problem

$$
[\tilde{\mathbf{W}} ; \tilde{\mathbf{Q}}]=\left[\begin{array}{c:c}
\mathbf{U}_{1} ; & \mathbf{K} \\
\mathbf{U}_{2} ; & \mathbf{G}_{0} \\
\mathbf{U}_{3} ; & \mathbf{G}_{1} \\
\mathbf{W} ; & \mathbf{Q}
\end{array}\right]
$$

So, the unknown Fibonacci coefficients are obtained as

$$
\mathbf{A}=(\tilde{\tilde{\mathbf{W}}})^{-1} \tilde{\tilde{Q}}
$$

where $[\tilde{\tilde{\mathbf{W}}} ; \tilde{\tilde{\mathbf{Q}}}]$ is generated by using the Gauss elimination method and then removing zero rows of the augmented matrix $[\tilde{\mathbf{W}} ; \tilde{\mathbf{Q}}]$.

\section{Error estimation algorithm and analyses}

In this section, we will give an efficient error estimation for the Fibonacci polynomial approximation and also a technique to obtain the corrected solution of the problem (1) by using the residual correction method (Oliveira 1980; Shahmorad 2005; Çelik 2006). For our aim, we define the residual function for the present method as

$$
R_{N}(x, t)=L\left[u_{N}(x, t)\right]-q(x, t)
$$

where

$$
L\left[u_{N}(x, t)\right]=\frac{\partial u_{N}(x, t)}{\partial x}-c(x) \frac{\partial^{\alpha} u_{N}(x, t)}{\partial x^{\alpha}} .
$$

Note that, Fibonacci polynomial solution satisfies the following problem

$$
L\left[u_{N}(x, t)\right]=\frac{\partial u_{N}(x, t)}{\partial x}-c(x) \frac{\partial^{\alpha} u_{N}(x, t)}{\partial x^{\alpha}}=q(x, t)+R_{N}(x, t)
$$

with the initial and boundary conditions

$$
\begin{array}{ll}
u_{N}(x, 0)=k(x), & 0<x<l \\
u_{N}(0, t)=g_{0}(t), & 0<t \leq \tau \\
u_{N}(l, t)=g_{1}(t), & 0<t \leq \tau .
\end{array}
$$

Furthermore, the error function $e_{N}(x, t)$ can be defined as

$$
e_{N}(x, t)=u(x, t)-u_{N}(x, t)
$$

where $u(x, t)$ is the exact solution of the problem (1). 
By using Eqs. (1) and (11-13), we have the error differential equation

$$
L\left[e_{N}(x, t)\right]=L[u(x, t)]-L\left[u_{N}(x, t)\right]=-R_{N}(x, t)
$$

with the homogenous conditions

$$
\begin{array}{ll}
e_{N}(x, 0)=0, & 0<x<l \\
e_{N}(0, t)=0, & 0<t \leq \tau \\
e_{N}(l, t)=0, & 0<t \leq \tau .
\end{array}
$$

Subsequently the error problem can be written as

$$
\begin{aligned}
& \frac{\partial e_{N}(x, t)}{\partial x}-c(x) \frac{\partial^{\alpha} e_{N}(x, t)}{\partial x^{\alpha}}=-R_{N}(x, t) \\
& e_{N}(x, 0)=0, \quad 0<x<l \\
& e_{N}(0, t)=0, \quad 0<t \leq \tau \\
& e_{N}(l, t)=0, \quad 0<t \leq \tau .
\end{aligned}
$$

Solving the problem (14) in the same way as in "The fractional derivative in the Caputo sense" section, we get the approximation $e_{N, M}(x, t)$ to $e_{N}(x, t), M>N$ which is the error function based on the residual function. We note that if the exact solution of the problem (1) is unknown, then the error function can be estimated by $e_{N, M}(x, t)$ which is found without the exact solution and also clearly seen from given error estimation algorithm. By means of the Fibonacci polynomial solution $u_{N}(x, t)$ and error estimation function $e_{N, M}(x, t)$, we obtain the corrected Fibonacci polynomial solution

$$
u_{N, M}(x, t)=u_{N}(x, t)+e_{N, M}(x, t) .
$$

\section{Numerical examples}

In this section, two examples are given to illustrate the applicability of the Fibonacci matrix method and all of them are performed on the computer by using MAPLE symbolic program.

Example 1 Consider the one-dimensional space fractional diffusion equation (Dehghan 2006)

$$
\frac{\partial u(x, t)}{\partial t}=c(x) \frac{\partial^{1.8} u(x, t)}{\partial x^{1.8}}+q(x, t), \quad 0<x<1, \quad 0<t \leq 2
$$

where $q(x, t)=\left(6 x^{3}-3 x^{2}\right) e^{-t}$, diffusion coefficient $c(x)=\Gamma(1.2) x^{1.8}$ with the initial condition

$$
u(x, 0)=x^{2}-x^{3}, \quad 0<x<1
$$

and the boundary conditions are

$$
\begin{aligned}
& u(0, t)=0 \\
& u(1, t)=0
\end{aligned}
$$

The exact solution of this problem $u(x, t)=\left(x^{2}-x^{3}\right) e^{-t}$.

Figure 1 shows the Fibonacci polynomial solution of this problem obtained by present method for $N=5$ with the exact solution. 


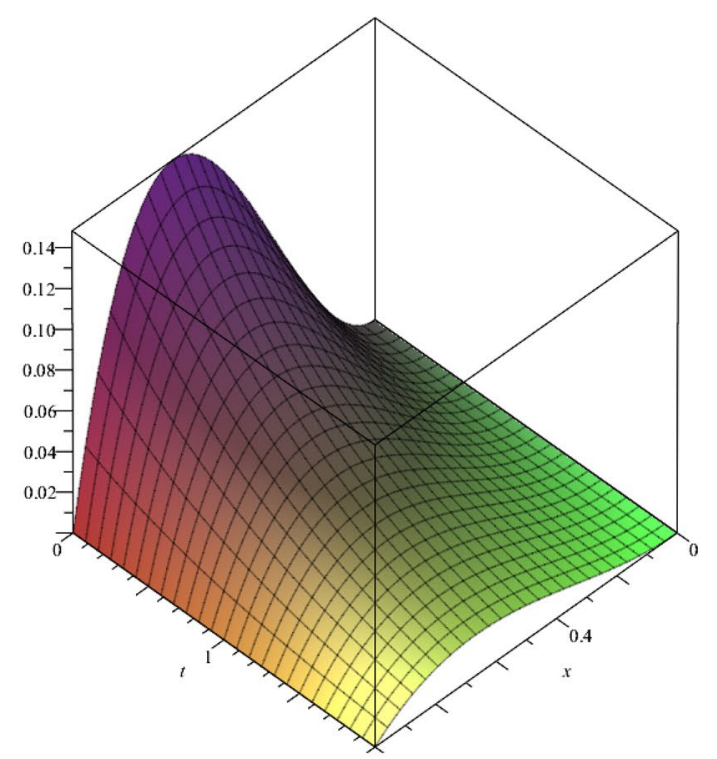

$\mathbf{a}$

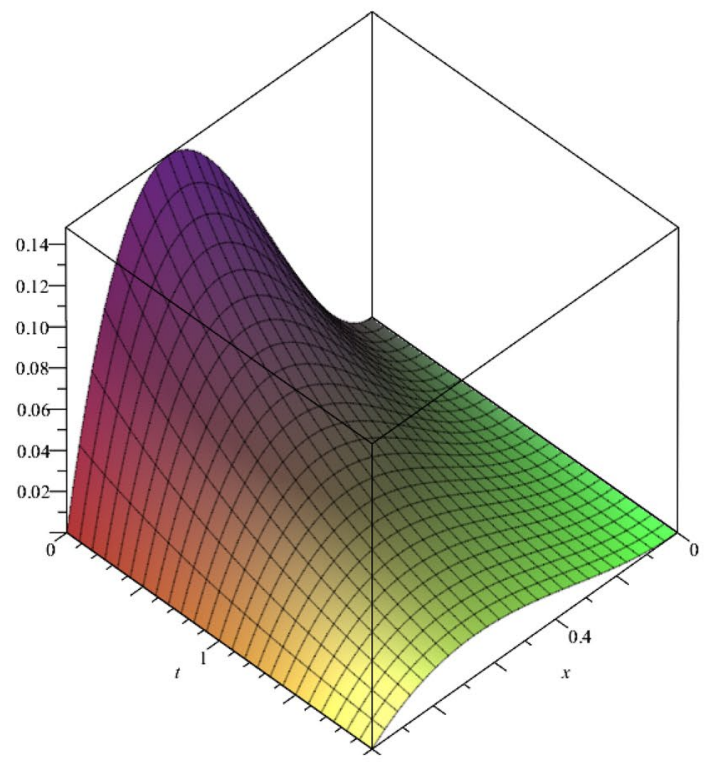

b

Fig. 1 Comparison of the exact solution (a) and Fibonacci polynomial solution for $N=5(\mathbf{b})$ of the Example 1

In Table 1, we have presented comparison of the absolute errors of the Fibonacci polynomial solution and improved Fibonacci polynomial solution that is found by using error estimation algorithm with finite difference method (Dehghan 2006) and Tau method (Saadatmandi and Dehghan 2011) for the same order polynomial solutions at $t=2$. We can clearly see both Fibonacci polynomial solution and improve Fibonacci 
Table 1 Comparison of the absolute error of the Fibonacci polynomial solution $\left(u_{N}(x, t)\right)$ and improved Fibonacci polynomial solution $\left(u_{N, M}(x, t)\right)$ with other methods for the same order polynomial solutions at $\boldsymbol{t}=\mathbf{2}$ for Example 1

\begin{tabular}{|c|c|c|c|c|}
\hline \multirow[t]{2}{*}{$x$} & \multirow{2}{*}{$\begin{array}{l}\text { Finite difference method } \\
\text { (Dehghan 2006) } \\
m=5\end{array}$} & \multirow{2}{*}{$\begin{array}{l}\text { Tau method } \\
\text { (Saadatmandi } \\
\text { and Dehghan 2011) }\end{array}$} & \multicolumn{2}{|c|}{ Present method } \\
\hline & & & $N=6$ & $N, M=6,7$ \\
\hline 0.1 & $4.20 e-5$ & $4.47 e-6$ & $1.35 e-6$ & $1.89 e-7$ \\
\hline 0.2 & $3.76 e-5$ & $2.78 e-7$ & $1.46 e-6$ & $4.01 e-7$ \\
\hline 0.3 & $8.44 e-5$ & $5.81 e-6$ & $4.09 e-7$ & $5.41 e-7$ \\
\hline 0.4 & $3.27 e-5$ & $1.02 e-5$ & $1.49 e-6$ & $6.02 e-7$ \\
\hline 0.5 & $3.61 e-5$ & $1.17 e-5$ & $3.76 e-6$ & $6.11 e-7$ \\
\hline 0.6 & $1.94 e-5$ & $1.08 e-5$ & $5.84 e-6$ & $5.92 e-7$ \\
\hline 0.7 & $2.95 e-5$ & $8.54 e-6$ & $7.07 e-6$ & $5.49 e-7$ \\
\hline 0.8 & $4.92 e-5$ & $6.06 e-6$ & $6.86 e-6$ & $4.58 e-7$ \\
\hline 0.9 & $2.83 e-5$ & $3.67 e-6$ & $4.65 e-6$ & $2.84 e-7$ \\
\hline
\end{tabular}

polynomial solution are better approximate than the other solution for the same order polynomial solution.

Figure 2 is indicated the absolute error function obtained by the our method for $N=7$, 11 and is clearly showed when $N$ is increased the absolute error values is decreased in the determined domain of the problem.

Example 2 In this example is the one-dimensional space fractional diffusion equation (Saadatmandi and Dehghan 2011)

$$
\frac{\partial u(x, t)}{\partial x}=c(x) \frac{\partial^{1.5} u(x, t)}{\partial x^{1.5}}+q(x, t), \quad 0<x<1, \quad 0<t \leq 1
$$

where $q(x, t)=\left(x^{2}+1\right) \cos (t+1)-2 x \sin (t+1)$, diffusion coefficient $c(x)=\Gamma(1.5) x^{0.5}$ with the initial condition

$$
u(x, 0)=\left(x^{2}+1\right) \sin (1), \quad 0<x<1
$$

and the boundary conditions are

$$
\begin{aligned}
& u(0, t)=\sin (t+1), \\
& u(1, t)=2 \sin (t+1) .
\end{aligned}
$$

The exact solution of this problem $u(x, t)=\left(x^{2}+1\right) \sin (t+1)$.

Figure 3 shows the absolute error functions $\left|u(x, t)-u_{\mathrm{N}}(x, t)\right|$ for the Fibonacci polynomial solutions for $N=8,12$. The absolute error values are decreased as $N$ is increased.

The absolute errors values have given the Fibonacci collocation method are compared with Tau method (Saadatmandi and Dehghan 2011) for $t=1$ and various values of first independent variable from 0.1 to 0.9 by 0.1 in Table 2 . And also each column has given the same truncated numbers for the Tau and present methods. 


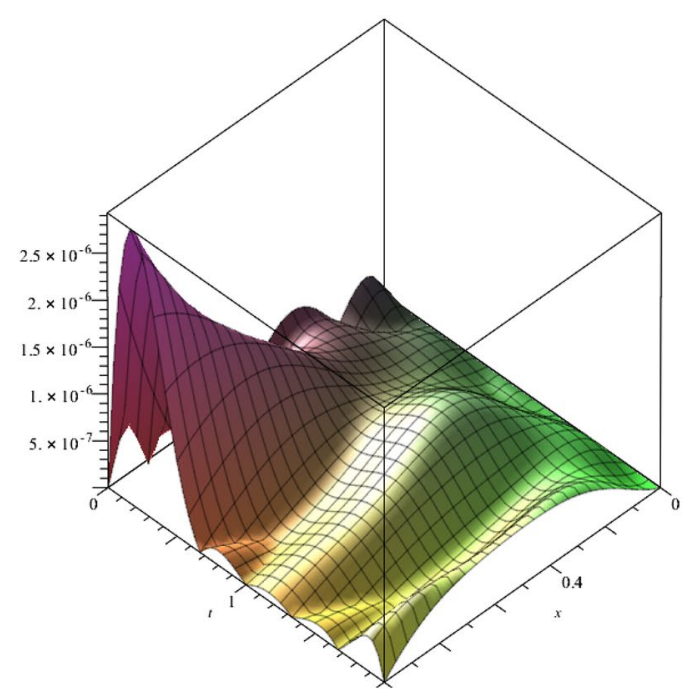

$\mathbf{a}$

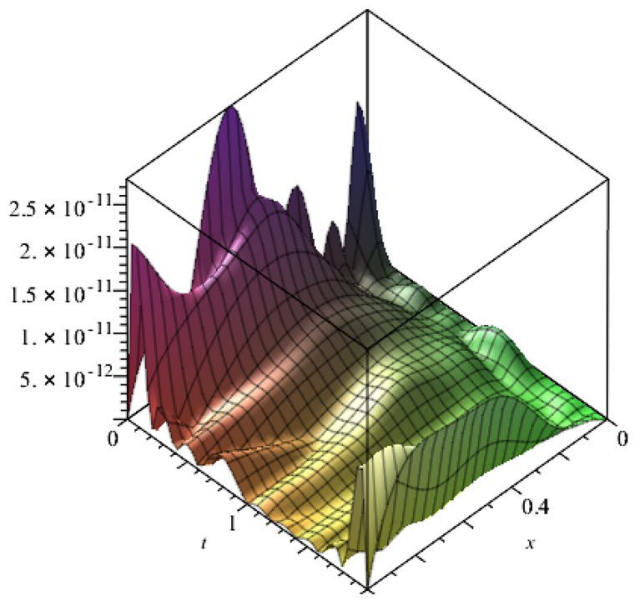

b

Fig. 2 Comparison the absolute error functions of Fibonacci polynomial solution for $N=7$ (a) and $N=11$ (b) for the Example 1

Figures 4 and 5 have been compared the absolute error function of $\left|e_{6}(x, t)\right|$ and error estimation functions $\left|e_{6,7}(x, t)\right|$ and $\left|e_{6,8}(x, t)\right|$ with respectively $t=1$ and $x=1$.

\section{Conclusion}

In this study, we have presented a new method using the Fibonacci polynomials to solve the one-dimensional space fractional diffusion equation. To this aim, we transformed the Fibonacci polynomials from algebraic form to matrix form. This method has been applied to two numerical examples which are indicated to illustrate the accuracy and efficiency. It can be observed from the results that the Fibonacci collocation method 


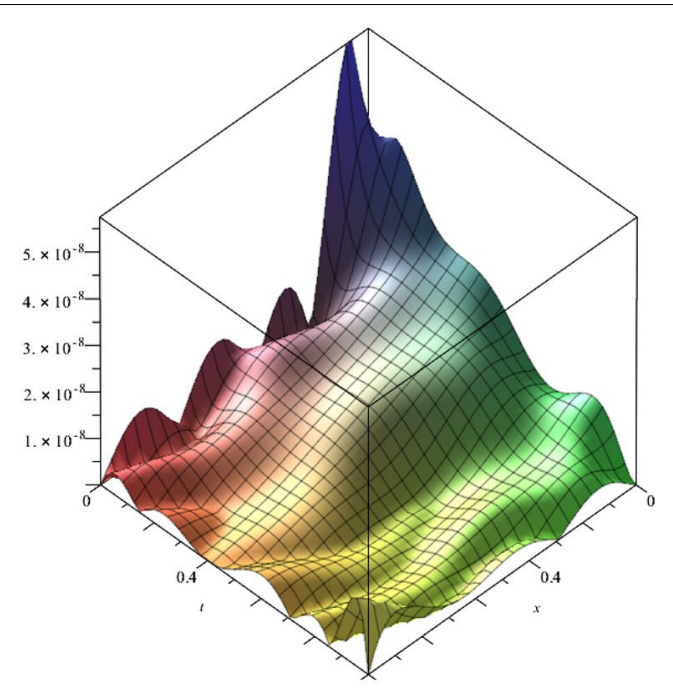

$\mathbf{a}$

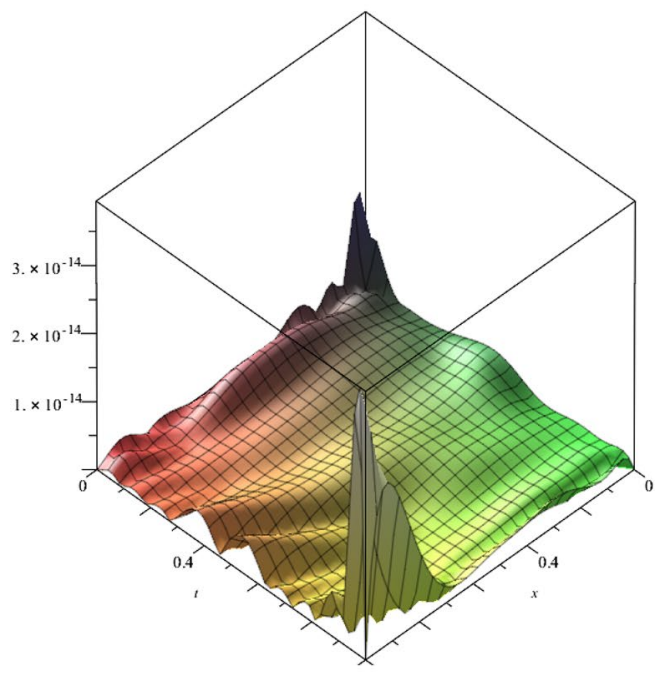

b

Fig. 3 Comparison the absolute error functions of Fibonacci polynomial solution for $N=8(\mathbf{a})$ and $N=12(\mathbf{b})$ for the Example 2

yields better approximation than the mentioned methods for the exact solution of the illustrated problems. It is observed from discussed examples which have the exact solution, the error estimation algorithm is very effective when the compared absolute errors of these examples. One of the considerable advantage of this method is that the approximate solutions are found very easily by using the symbolic programs. 
Table 2 Comparison of the absolute error of the $u_{N}(x, t)$ with other methods for $t=1$ for the Example 2

\begin{tabular}{lllll}
\hline $\boldsymbol{x}$ & $\begin{array}{l}\text { Tau method } \\
\text { (Saadatmandi and } \\
\text { Dehghan 2011) } \\
\boldsymbol{m}=\mathbf{6}\end{array}$ & Present method & $\begin{array}{l}\text { Tau method } \\
\text { (Saadatmandi } \\
\text { and Dehghan 2011) } \\
\boldsymbol{m}=\mathbf{7}\end{array}$ & Present method \\
\hline 0.1 & $5.35 e-5$ & $4.66 e-5$ & $9.81 e-7$ \\
0.2 & $1.11 e-4$ & $4.19 e-6$ & $7.74 e-5$ & $1.60 e-6$ \\
0.3 & $1.19 e-4$ & $1.11 e-6$ & $5.00 e-5$ & $1.64 e-6$ \\
0.4 & $7.65 e-5$ & $1.89 e-6$ & $2.30 e-5$ & $1.69 e-6$ \\
0.5 & $4.06 e-5$ & $4.40 e-6$ & $2.74 e-5$ & $1.58 e-6$ \\
0.6 & $3.30 e-5$ & $6.04 e-6$ & $4.38 e-5$ & $1.34 e-6$ \\
0.7 & $4.42 e-5$ & $6.74 e-6$ & $3.87 e-5$ & $1.11 e-6$ \\
0.8 & $5.38 e-5$ & $6.46 e-6$ & $1.01 e-5$ & $9.92 e-7$ \\
0.9 & $2.79 e-5$ & $5.20 e-6$ & $3.35 e-6$ & $8.73 e-7$ \\
\hline
\end{tabular}

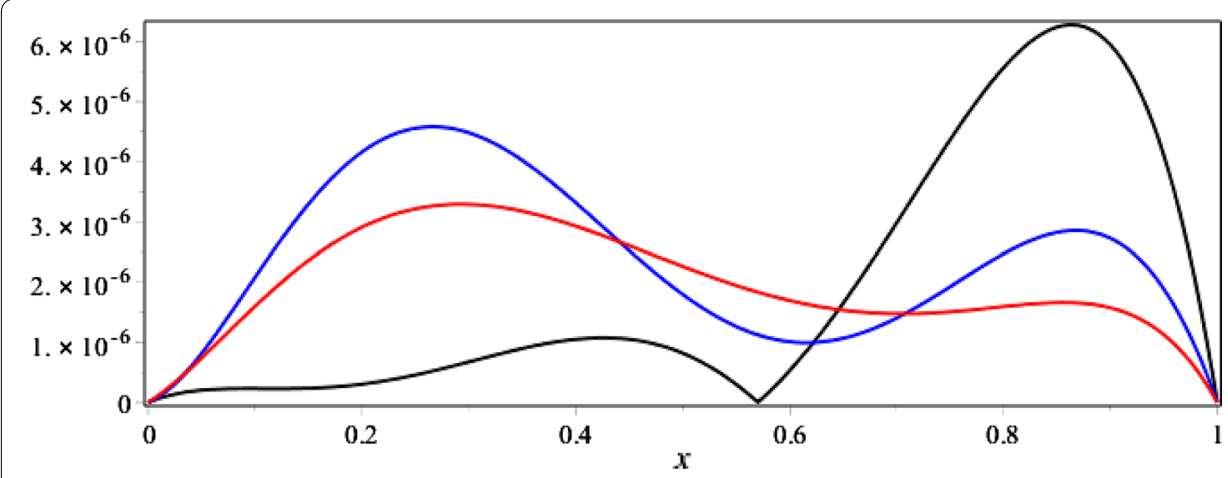

Fig. 4 The comparison of the absolute error function $\left|e_{6}(x, 1)\right|($ black $)$ and error estimation functions $\left|e_{6,7}(x, 1)\right|(b / u e)$ and $\left|e_{6,8}(x, 1)\right|($ red $)$ for the Example 2

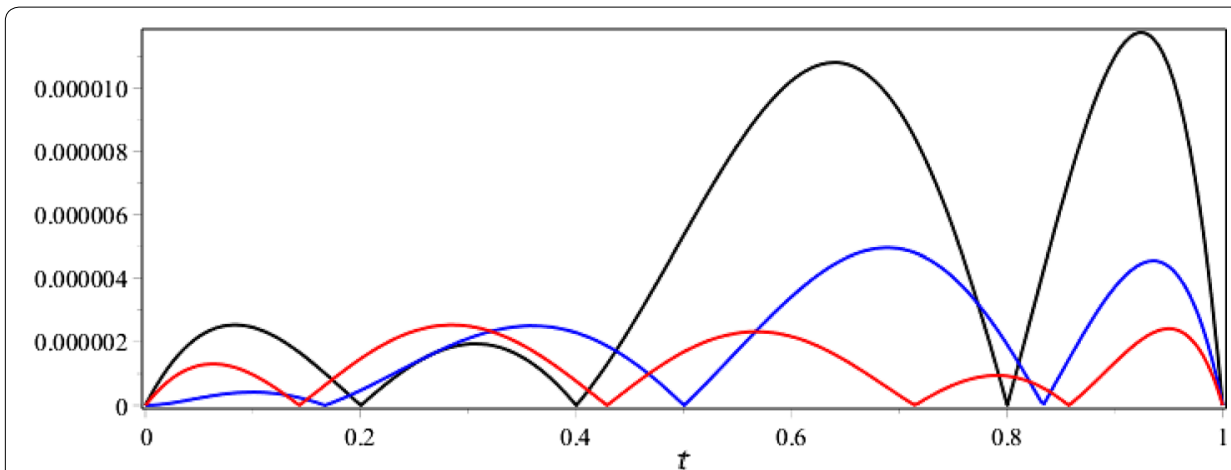

Fig. 5 The comparison of the absolute error function $\left|e_{6}(1, t)\right|$ (black) and error estimation functions $\left|e_{6,7}(1, t)\right|($ blue $)$ and $\left|e_{6,8}(1, t)\right|($ red $)$ for the Example 2 


\section{Authors' contributions}

AKB and SY collectively carried out this study, improved method, and writing software them. AKB wrote the manuscript. SY helped to revise the manuscript. Both authors read and approved the final manuscript.

\section{Competing interests}

The authors declare that they have no competing interests.

Received: 16 May 2016 Accepted: 18 July 2016

Published online: 22 August 2016

\section{References}

Bülbül B, Sezer M (2011) A Taylor matrix method for the solution of a two-dimensional linear hyperbolic equation. Appl Math Lett 24(10):1716-1720

Çelik I (2006) Collacation method and residual correction using Chebyshev series. Appl Math Comput 174:910-920

Çevik M (2010) Application of Taylor matrix method to the solution of longitudinal vibration of rods. Math Comput Appl 15(3):334-343

Dehghan M (2006) Finite difference procedures for solving a problem arising in modeling and design of certain optoelectronic devices. Math Comput Simul 71:16-30

Diethelm K (2010) The analysis of fractional differential equations. Springer, Berlin

Dönmez Demir D, Bildik N, Sınır BG (2013) Linear vibrations of continuum with fractional derivatives. Bound Value Probl 104:1-15

Erdem Biçer K, Yalçınbaş S (2016) A matrix approach to solving hyperbolic partial differential equations using Bernoulli polynomials. Filomat 30(4):993-1000

Isik OR, Sezer M, Güney Z (2014) Bernstein series solution of linear second-order partial differential equations with mixed conditions. Math Methods Appl Sci 37(5):609-619

Khader MM (2011) On the numerical solutions for the fractional diffusion equations. Commun Nonlinear Sci Numer Simul 16:2535-2542

Kurt Bahşı A, Yalçınbaş S (2016a) A new algorithm for the numerical solution of telegraph equations by using Fibonacci polynomials. Math Comput Appl 21-2(15):1-12

Kurt Bahşı A, Yalçınbaş S (2016b) Fibonacci collocation method with a residual error function to solve linear Volterra integro differential equations. NTMSCI 4(1):1-14

Kurt Bahşı A, Şahin N, Sezer M (2015) A numerical algorithm with residual error estimation for solution of high-order Pantograph-type functional differential equations using Fibonacci polynomials. NTMSCI 3(3):90-102

Kurt A, Yalçınbaş S, Sezer M (2013a) Fibonacci collocation method for solving linear differential-difference equations. Math Comput Appl 18(3):448-458

Kurt A, Yalçınbaş S, Sezer M (2013b) Fibonacci collocation method for solving high-order linear Fredholm integro-differential-difference equations. Dig J Int J Math Sci. doi:10.1155/2013/486013

Oliveira FA (1980) Collacation and residual correction. Numer Math 36:27-31

Saadatmandi A, Dehghan M (2011) A tau approach for solution of the space fractional diffusion equation. Comput Math Appl 62:1135-1142

Shahmorad S (2005) Numerical solution of general form linear Fredholm. Volterra integro differantial equations by the tau method with an error estimation. Appl Math Comput 167:1418-1429

Tadjeran C, Meerschaert MM, Scheffler HP (2006) A second order accurate numerical approximation for the fractional diffusion equation. J Comput Phys 213:205-213

Yüksel G, Sezer M (2014) A Chebyshev series approximation for linear second-order partial differential equations with complicated conditions. Gazi Univ J Sci 26(4):515-525

Yüzbaşı Ş, Şahin N (2013) Numerical solutions of singularly perturbed one-dimensional parabolic convection-diffusion problems by the Bessel collocation method. Appl Math Comput 220:305-315

\section{Submit your manuscript to a SpringerOpen ${ }^{\circ}$ journal and benefit from:}

- Convenient online submission

- Rigorous peer review

- Immediate publication on acceptance

- Open access: articles freely available online

- High visibility within the field

- Retaining the copyright to your article

Submit your next manuscript at springeropen.com 